\title{
AN INVESTIGATION ON OIL/WATER SEPARATION MECHANISM INSIDE HELICAL PIPES
}

Zhang jun, Guo jun, Gong Dao-tong, Wang Li-yang, Tang Chi, Zheng Zhi-chu Institute of Mechanics, Chinese Academy of Sciences, Beijing, 100080, China

E-mail: zhangjun@imech.ac.cn

\begin{abstract}
Helical pipes are the core components of combination-type oil/water separator. Study on oil/water separation mechanism inside helical pipes was conducted through numerical simulation and experiments. The modified design based on the research has been successfully applied in highefficiency oil/water separator.
\end{abstract}

KEY WORDS: helically pipe, oil/water separation, experimental studies, numerical simulation.

\section{Introduction}

It is an effective way to apply several separation principles synthetically in order to achieve high-efficiency separation ${ }^{[1]}$. Therefore, a kind of efficient separator (Fig. 1) integrated with several separation principles, including centrifugal force, gravitational sedimentation, expansion and vibration, was put forward.

To meet separation requirements, all components of the combination-type separator must be tested, of which the helical pipes are the most important. Consequently, oil/water separating process is investigated through both experimental sampling and numerical simulation in order to investigate the mechanism of oil/water separation.

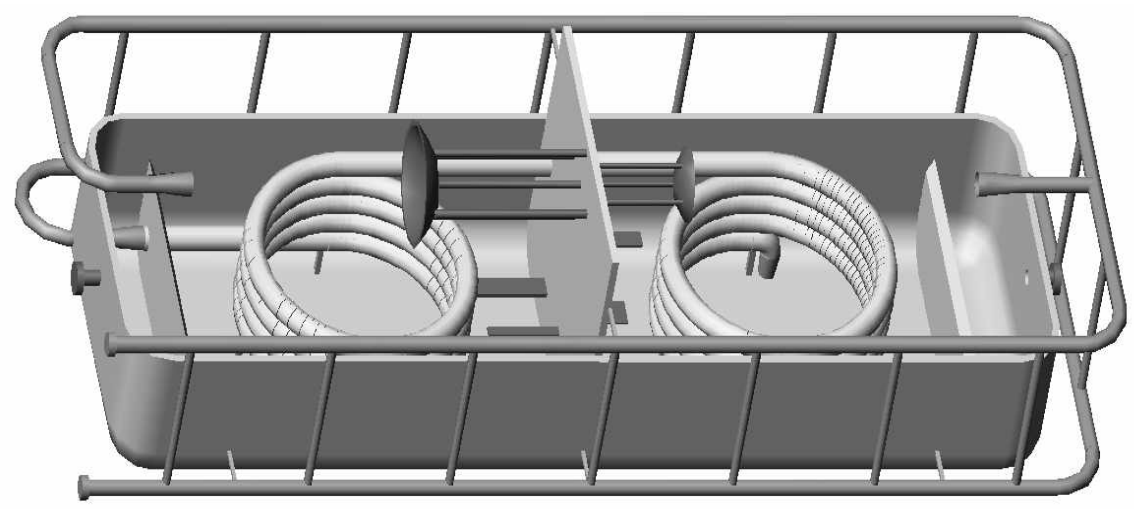

\section{Fundamental Equations of Oil/Water Flow}

The Euler-Euler method and the Euler model are applied to conduct the numerical simulation, and a discrete phase model to describe the two-phase flow inside the separator ${ }^{[2-10]}$.

\subsection{Continuity equations}

The continuity equations for oil and water are given as

$$
\nabla \cdot\left(\alpha_{q} \rho_{q} \vec{v}_{q}\right)=0, \quad(q=1,2)
$$

\subsection{Momentum equation}

The momentum equation for phase $\boldsymbol{q}$ is expressed as

$$
\nabla \cdot\left(\partial_{q} \rho_{q} \bar{v}_{p} \bar{v}_{p}\right)=-\partial_{q} \nabla p+\nabla \cdot \overline{\tau_{q}}+\partial_{q} \rho_{q} \bar{g}+k_{m}\left(\bar{v}_{p}-\overline{v_{q}}\right)
$$

where, $\overline{\overline{\tau_{q}}}$ is defined as

$$
\overline{\overline{\tau_{q}}}=\alpha_{q} \mu_{q}\left(\nabla \vec{v}_{q}+\nabla \overrightarrow{v_{q}^{T}}\right)+\alpha_{q}\left(\lambda_{q}-\frac{2}{3} \mu_{q}\right) \nabla \cdot \vec{v}_{q} \overline{\bar{I}}
$$

And $K_{p q}$ depends on friction, pressure, cohesion, etc. The Inter-phase change coefficients are calculated using the Morsi-Alexander model, that is, the secondary (dispersed) phase is in the form of liquid 
droplets.

$K_{p q}$ can be written in the following form:

$K_{p q}=\frac{3 \mu_{q} \alpha_{p} \mathrm{Re}}{4 d_{p}{ }^{2}}\left(a_{1}+\frac{a_{2}}{\mathrm{Re}}+\frac{a_{3}}{\mathrm{Re}^{2}}\right)$

$\operatorname{Re}=\frac{\rho_{q}\left|\vec{v}_{p}-\vec{v}_{q}\right| d_{p}}{\mu_{q}}$

$a_{1}, a_{2}, a_{3}=$

$\begin{cases}0,18,0 & 0<\operatorname{Re}<0.1 \\ 3.690,22.73,0.0903 & 0.1<\operatorname{Re}<1 \\ 1.222,29.1667,-3.8889 & 1<\operatorname{Re}<10 \\ 0.6167,46.50,-116.67 & 10<\operatorname{Re}<100 \\ 0.3644,98.33,-2778 & 100<\operatorname{Re}<1000 \\ 0.357,148.62,-47500 & 1000<\operatorname{Re}<5000 \\ 0.46,-490.546,578700 & 5000<\operatorname{Re}<10000\end{cases}$

\subsection{Turbulence model}

The $k-\varepsilon$ model is selected in the turbulence calculation.

$$
\begin{aligned}
& \nabla \cdot\left(\rho_{m} \vec{v}_{m} k\right)=\nabla \cdot\left(\frac{\mu_{t, m}}{\sigma_{k}} \nabla k\right)+G_{k, m}-\rho_{m} \varepsilon \\
& \nabla \cdot\left(\rho_{m} \vec{v}_{m} \varepsilon\right)=\nabla \cdot\left(\frac{\mu_{t, m}}{\sigma_{\varepsilon}} \nabla \varepsilon\right)+\frac{\varepsilon}{k}\left(G_{1 \varepsilon} G_{k, m}-G_{2 \varepsilon} \rho_{m} \varepsilon\right)
\end{aligned}
$$

where,

$$
\begin{aligned}
& \rho_{m}=\sum_{i=1}^{N} \alpha_{i} \rho_{i} \\
& \vec{v}_{m}=\sum_{i=1}^{N} \alpha_{i} \rho_{i} \vec{v}_{i} / \sum_{i=1}^{N} \alpha_{i} \rho_{i} \\
& G_{k, m}=\mu_{t, m}\left[\nabla \vec{v}_{m}+\left(\nabla \vec{v}_{m}\right)^{\mathrm{T}}\right]: \nabla \vec{v}_{m} \\
& \mu_{t, m}=\rho_{m} C_{\mu} \frac{k^{2}}{\varepsilon}
\end{aligned}
$$

\subsection{Boundary conditions}

Boundary conditions should be set for inlet, outlet and wall. In the present paper, all physical variables are given at the inlet and supposed to be distributed uniformly. The static pressure is considered to be atmospheric pressure $\left(1.01325 \times 10^{5} \mathrm{~Pa}\right)$. Fully developed flow is defined for outlet boundary, indicating that normal gradients of all physical variables are negligible. The back-pressure is atmospheric pressure. No-slip boundary condition is enforced for walls, meaning both the velocity and turbulent intensity are zero there.

\section{Numerical Methods}

The commercial CFD software, FLUENT, is used to simulate the oil/water separation, where the SIMPLE algorithm based on finite element is applied [11].

\subsection{Creation of finite element}

The volume cells are created using the Gambit. Firstly, a profile (circle) with a distance of $\triangle \mathrm{Z}$ along the axes is meshed using a trianglular grid, which becomes denser from the center to wall surface in order to capture the characteristics of boundary layer. Then, the corresponding points within all profiles are connected to form triangular cells.

\subsection{Discrete form of fundamental equations}

All conservation equations can be expressed in one form, namely convective term is the sum of diffusion term and source term in the SIMPLE algorithm. For each cell, a general discrete form of equation for $\varphi$ at $\mathrm{P}$ can be expressed as

$a_{P} \varphi_{P}=a_{E} \varphi_{E}+a_{W} \varphi_{W}+a_{N} \varphi_{N}+a_{S} \varphi_{S}+a_{T} \varphi_{T}+a_{B} \varphi_{B}+b$

where, $\alpha$ and $b$ are the coefficients, while the subscript $P$ represents the calculated node, and $E, W$, $N, S, T, B$ are the adjacent surrounded nodes.

\subsection{Numerical algorithm}

A modified $\mathrm{P}-\mathrm{V}$ discrete algorithm is applied to solve the discrete equations ${ }^{[12]}$.

\section{Calculation and Analysis}

Parameters in the calculation are as follows: the oil density $836 \mathrm{~kg} / \mathrm{m}^{3}$, the oil viscosity $20 \mathrm{mPa} \cdot \mathrm{s}$; water density $998.2 \mathrm{~kg} / \mathrm{m}^{3}$, the water viscosity 1.00131 $\mathrm{mPa} \cdot \mathrm{s}$; the gravitational acceleration $9.81 \mathrm{~m} / \mathrm{s}^{2}$.

\subsection{Oil/water separation inside helical pipes without outflow}

The geometry of helical pipe is shown in Fig. 2: the pipe diameter $D=40 \mathrm{~mm}$, the gyration radius $R=400 \mathrm{~mm}$, the pitch of screws $t=100 \mathrm{~mm}$. The number of tubes can be increased according to specific conditions. The water cut at the inlet is 30 percent. The oil/water mixture enters the inlet at the bottom of helical pipe, and flows out at the top. To investigate the influence of water droplet size on separation, three mean diameters, namely $d=0.1 \mathrm{~mm}, 2 \mathrm{~mm}, 5 \mathrm{~mm}$, are selected in the calculation. The volume fraction of oil on the profile at an angle of $\theta=270$ to the starting point of the $5^{\text {th }}$ helically coiled tube is shown in Fig. 3, while the calculated results for $d=0.3 \mathrm{~mm}$ and $d=2 \mathrm{~mm}$ in Fig. $3 \sim 4$.

Through the comparison between simulated results, it can be found that the mean water droplet sizes have an obvious influence on the final separation. The larger the droplet diameter, the less time the oil/water mixture needs for separation. In the case of $d=5 \mathrm{~mm}$, the lower part of the tube profile is dominated with water after only one circle. While it takes much longer time to separate as the mean droplet size is set as $d=0.1 \mathrm{~mm}$. 


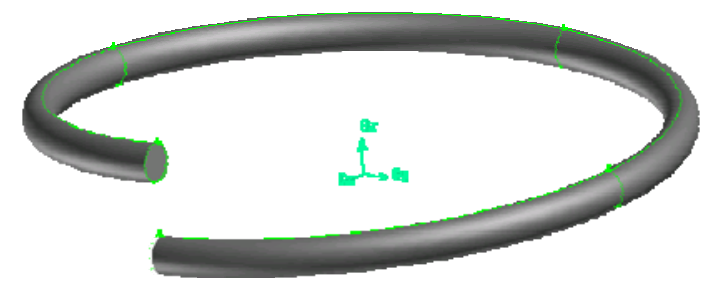

Fig. 2 Geometry of helicallycoled tubes
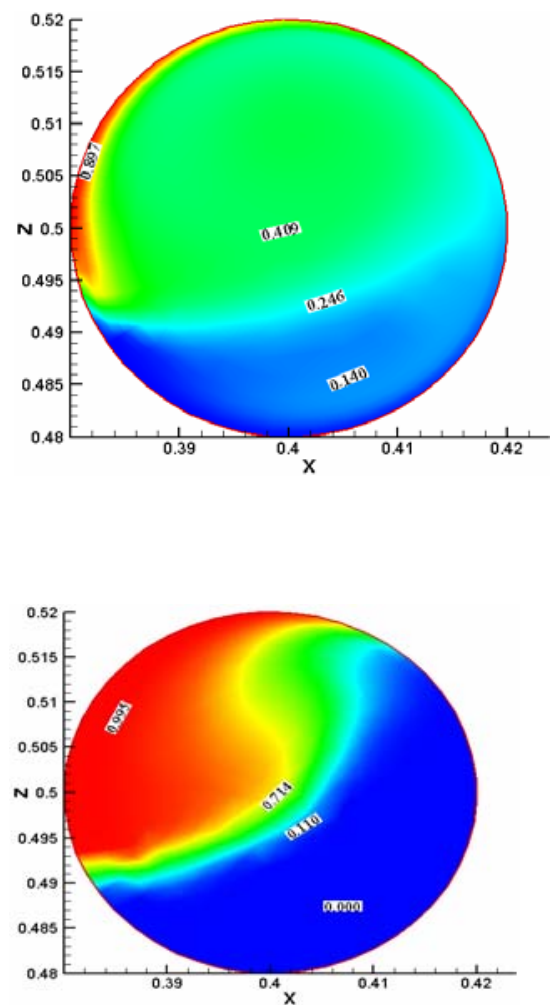

Fig. 4 Volume fraction distribution on the profile of the fifth tube $(d=2.0 \mathrm{~mm})$

\subsection{Oil/water separation inside helical pipes with outflow through small holes}

Based on the simulation results, as well as many experiments, the design of helical pipes was modified. The gyration radius was reduced from $400 \mathrm{~mm}$ to 150 $\mathrm{mm}$ to increase the centrifugal force, and some small holes were drilled at proper positions on the tubes for the purpose of discharging part of water. The oil/water distribution after one hole is defined as the initial conditions for the next one, and in this way the recursion calculation is continued.

In this designed model, the helical pipes are placed vertically. The internal diameter of tube $d=$ $22 \mathrm{~mm}$, and the pitch of screws $h=53 \mathrm{~mm}$. Totally there are 15.5 circles of tubes. There is no outflow from the $1^{\text {st }}$ to $11^{\text {th }}$ circles, while small holes appear from the $12^{\text {th }}$ one. Thus, the holes are distributed uniformly along the final three circles, of which interval angle between the adjacent holes is $180^{\circ}$. Three diameters are designed for these holes, namely, $3 \mathrm{~mm}, 2.5 \mathrm{~mm}$ and $2.5 \mathrm{~mm}$, respectively. The holes are marked from 1 to 18 in order. At the inlet, the mixture velocity $v=0.797 \mathrm{~m} / \mathrm{s}$, the gauge pressure $0.17 \times 10^{6}$ $\mathrm{MPa}$, and the oil volume fraction $43.89 \%$. The simulated results are shown in Fig. 5.
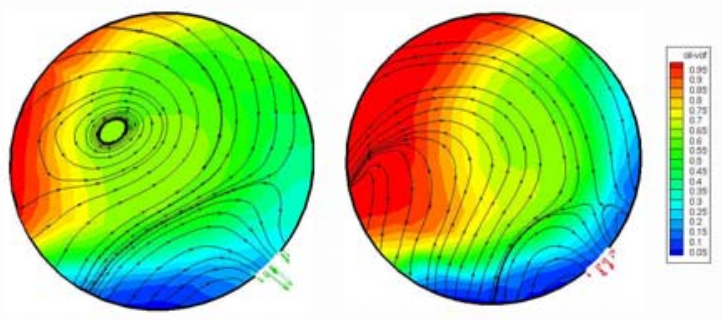

Fig. 5 Oil/water volume fraction distribution at the $9^{\text {th }}$ and $15^{\text {th }}$ holes

The simulated results indicate that the oil volume fraction is 63.9 percent at the outlet of helical pipes. The flow field inside tubes becomes more complex, as there exists outflow through the small holes. As the gyration radius decreases, water tends to accumulate in the right lower part of tubes. The volume fraction of oil phase gradually increases with the outflow of water phase.

\section{Separation Experiments of Helical Pipes}

We conducted experiments to verify the results of calculation. The main pipe used in the test rig is $50 \mathrm{~mm}$ i.d. and $15 \mathrm{~m}$ long, inside which the maximum velocity of gas can arrive at $50 \mathrm{~m} / \mathrm{s}$, and the maximum velocity of liquid $2 \mathrm{~m} / \mathrm{s}$. Several flow patterns, as well as mixture flow of various oil/water and oil/water/gas ratio, can be simulated. The layout of the experimental facilities is shown in Fig. 6.

Initially, the small holes were drilled at the outside center of tubes. However, both the experiments and simulated results indicate that it is not the optimal position for holes due to the gravitational acceleration. The holes should be placed where the water cut is highest. Thus, the design was modified according to experimental and simulated results, as shown in Fig. 7.

White oil and water are used as test medium in the experiment, which was conducted for both the two designed helical pipes (No. 1: the gyration radius $400 \mathrm{~mm}$, tube i.d. $40 \mathrm{~mm}$; No. 2: the gyration radius $150 \mathrm{~mm}$, tube i.d. $25 \mathrm{~mm}$ ), as shown in Fig. 8. The oil includes two types, namely LP- 14 (at $20^{\circ} \mathrm{C}$, density $836 \mathrm{~kg} / \mathrm{m}^{3}$, viscosity $20 \mathrm{mPa} \bullet \mathrm{s}$ ) and PS (at $20^{\circ} \mathrm{C}$, density $874 \mathrm{~kg} / \mathrm{m}^{3}$, viscosity $\left.70 \mathrm{mPa} \bullet \mathrm{s}\right) . \mathrm{K}_{2} \mathrm{MnO}_{4}$ is dissolved in water as tracer to distinguish visually the white oil and water. Water cut in oil/water mixture is from 10 percent to 90 percent. 


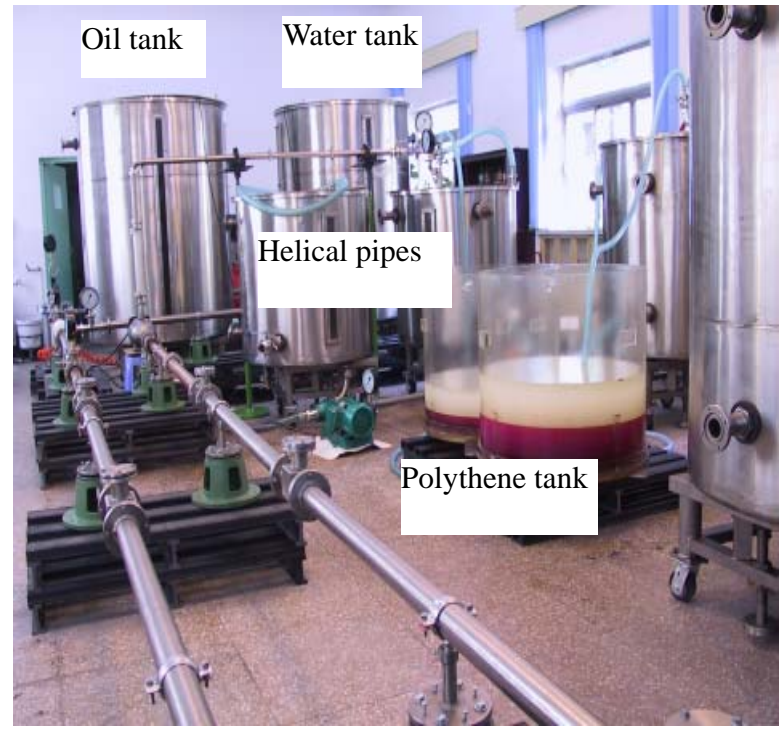

Fig. 6 Experimental facilities of helically coiled tubes

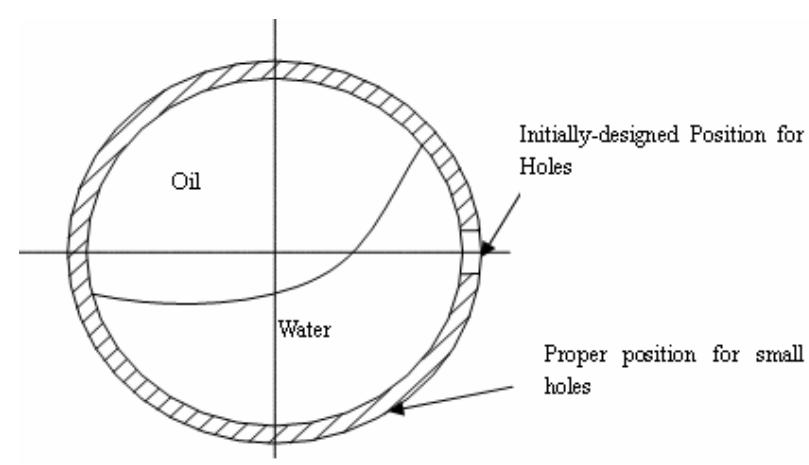

Fig. 7 Schematic diagram of position for small holes

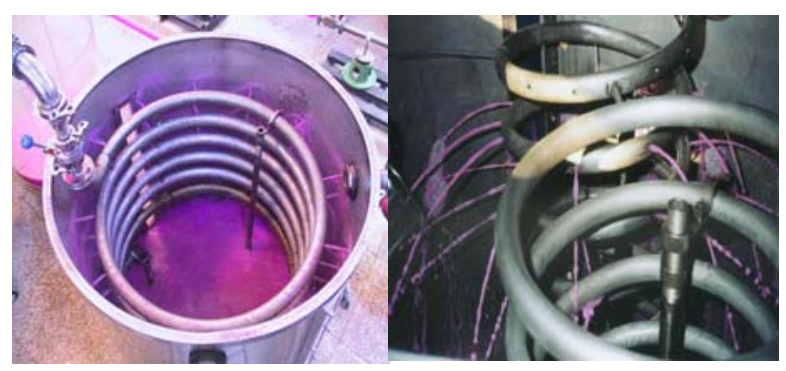

Fig. 8 Flow inside helically coiled tubes

Part of typical experimental results are given in Table 1 , where the flux and water cut for $1^{\text {st }}$ row to $3^{\text {rd }}$ one represent sampling results from small holes on corresponding circles.

In No. 1, the water cut along the tubes varies gently due to low viscosity of LP-14 white oil, high flow rate, and jet disturbance at the small holes. Oil and water are not separated well.

In No. 2, despite high flow rate, separation is improved due to larger centrifugal force of about $7 \mathrm{~g}$. Water tends to accumulate at the small holes, and therefore, the water cut at the outlet is decreased significantly, which is approximately 22 percent.

In No. 3, the flow rate inside tubes declines dramatically because too many holes are drilled along the tubes. Although the water cut falls about 80 percent, the flux is only 0.098 , which obviously cannot meet the requirement of practical engineering.

\section{Conclusions}

The following conclusions can be reached from the above description.

(1) The separation efficiency of helical pipes is the function of gyration radius, residence time, pressure at the inlet and outlet, density difference between phases, liquid droplet diameter, etc;

(2) The numerical simulation agrees well with the experimental results, proving the accuracy of mathematical model and indicating that it is reasonable to combine numerical simulation and experiments to design and modify helically coiled separators;

(3) For the separation of helical pipes, the centrifugal force is directly proportional to the square of flow rate inside tubes, while inversely proportional to the gyration radius. Thus, increasing flow rate and reducing gyration radius can effectively improve separation efficiency, both of which, however, are restricted due to machining techniques. At the same time, high flow rate means high-pressure drop, and the enhanced turbulent disturbance can affect negatively the separating process.

(4) Increasing the number of circles while reducing the gyration radius can reasonably prolong the flow time of oil/water mixture along tubes, which can improve separation significantly.

(5) The present helical pipes can separate water from oil/water mixture to a certain extent. Through numerical simulation and experiments, the separating process inside tubes was studied and optimal parameters were obtained, based on which new high-efficiency helical pipes can be designed.

(6) Helical pipes are the core component of threephase separators, inside which water separated from oil/water mixture would be introduced through small holes. The jet flux and water cut reveal the efficiency of helical pipes. 
Table 1

\begin{tabular}{|c|c|c|c|c|c|c|c|c|c|c|}
\hline \multirow[b]{2}{*}{ NO. } & \multirow[b]{2}{*}{ Oil } & \multirow[b]{2}{*}{$\begin{array}{c}\text { Gyration } \\
\text { radius }\end{array}$} & \multirow{2}{*}{$\begin{array}{c}\text { Inlet } \\
\text { velocity } \\
(\mathrm{m} / \mathrm{s})\end{array}$} & \multicolumn{2}{|c|}{ Flux $\left(\mathrm{m}^{3} / \mathrm{h}\right)$} & \multicolumn{5}{|c|}{ Water cut } \\
\hline & & & & Inlet & Outlet & Inlet & $\begin{array}{l}1^{\text {st }} \\
\text { row }\end{array}$ & $\begin{array}{l}2^{\text {nd }} \\
\text { row }\end{array}$ & $\begin{array}{l}3^{\text {rd }} \\
\text { row }\end{array}$ & outlet \\
\hline 1 & LP-14 & 400 & 1.59 & 7.18 & 4.83 & 0.644 & 0.649 & 0.637 & 0.676 & 0.642 \\
\hline 2 & LP-14 & 150 & 3.08 & 5.45 & 0.63 & 0.431 & 0.462 & 0.440 & 0.396 & 0.338 \\
\hline 3 & PS & 400 & 1.16 & 5.24 & 0.11 & 0.466 & 0.523 & 0.508 & 0.382 & 0.098 \\
\hline 4 & PS & 150 & 0.78 & 1.37 & 0.02 & 0.398 & 0.547 & 0.436 & 0.217 & 0.050 \\
\hline
\end{tabular}

\section{NOMENCLATURE}

\begin{tabular}{|c|c|c|c|}
\hline$\vec{g}$ & _ Gravitational acceleration & $d$ & —Particle diameter \\
\hline$\alpha$ & _ Volume fraction & $\mu_{t, m}$ & —_ Turbulence viscosity \\
\hline$\rho$ & —Density & Subscript $\boldsymbol{p}$ & —_Liquid phase $\boldsymbol{p}$ \\
\hline$\vec{v}$ & __ Velocity & Subscript $\boldsymbol{q}$ & —_iquid phase $\boldsymbol{q}$ \\
\hline $\bar{\tau}_{q}$ & — Shear stress tensor & $\mu_{q}$ & — Shear viscosity for phase $\boldsymbol{q}$ \\
\hline$K_{p q}$ & $\begin{array}{l}\text {-Interphase momentum exchange } \\
\text { coefficient }\end{array}$ & $\lambda_{q}$ & —Volume viscosity for phase $\boldsymbol{q}$ \\
\hline$n$ & _Pressure & $G_{k, m}$ & __ Turbulence kinetic energy \\
\hline$\rho_{m}$ & —Mixture density & $\sigma$ & __ Prandtl number \\
\hline$\vec{v}_{m}$ & —Mixture velocity & $\tau$ & __ Particle relaxation time \\
\hline
\end{tabular}

\section{References}

[1] ZHENG Zhi-chu, ZHOU Yong, GUO Jun, ZHANG Jun, TANG Chi. Separation technique for oil-gas multi-phase flow transportation in submarine pipeline[C]. Hong Kong. Proceedings of the 17th National Conference on Hydrodynamics and the 6th National Congress on Hydrodynamics, 2004, 830-837.

[2] T.B.Anderson , R.Jackson . A Fluid Mechanical Description of Fluidized Beds[J]. I \& EC Fundam, 1967(6), 527-534.

[3] R.M.Bowen . Theory of Mixtures . Continuum Physics[M]. New York: Academic Press, 1976, 1-127.

[4] Li Ran, Li Hong, Li Jia,Li Ke-feng. Application of gas-liquid two-phase theory for the water surface calculation in open channels. Journal of Hydrodynamics, Ser. A, 2002, 17(1), 77-83. (in Chinese)

[5] Diao Ming-jun, Yang Yong-quan, Xu Wei-lin, Wang Wei, Liu Shan-jun. Numerical simulation on 2-D water-air two-phase flow over top outlet[J] . Journal of Hydrodynamics, Ser.B, 2002, 14(3), 60-63.

[6] Yuri V. Fairuzov. Numerical simulation of transient flow of two immiscible liquids in pipeline[J], AIChE Journal, 2000, 46(7), 1332-1339.

[7] L.Schiller, Z.Naumann. Z. Ver .Deutsch. Ing.[M], 1935(77), 318.

[8] D.A.Drew , R.T.Lahey . Particulate Two-Phase Flow [M]. Boston: Butterworth-Heinemann, 1993, 509-566.

[9] CHEN Guo-qian, LI Xing-wei. Numerical experiment of two-dimensional line thermal .Journal of Hydrodynamics, Ser. A, 2000, 15(4), 411-423.

[10] B.E.Launder, D.B.Spalding. Lectures in Mathematical Models of Turbulence[R]. London: Academic Press, 1972.

[11] J.P.Vandoormaal, G.D.Raithby. Enhancements of the SIMPLE Method for Predicting Incompressible Fluid Flows. in Heat Transfer [M], 1984(7), 147-163.

[12] J.L.Ferzieger, M.Peric. Computational Methods for Fluid Dynamics[M]. Heidelberg: Springer-Verlag, 1996. 